\title{
Síndrome de Ortner asociado a estenosis aórtica grave, reporte de caso y revisión de la literatura
}

\author{
Ortner syndrome associated to severe aortic stenosis, case report and review \\ of the literature
}

\author{
Jairo A. Rentería-Roa ${ }^{1}$, Caren M. Ton-Mazo², Daniel Cardona-Correa ${ }^{3^{*}}$ y Cristian A. Piedrahita ${ }^{3}$ \\ ${ }^{1}$ Departamento de Cuidados Intensivos, IPS Universitaria Clínica León XIII; ${ }^{2}$ Medicina General, IPS Artmedica; ${ }^{3}$ Departamento de Cardiología, \\ IPS Universitaria Clínica León XIII. Medellín, Antioquia, Colombia
}

En 1897 Norbert Ortner describió tres pacientes con crecimiento atrial izquierdo, por estenosis valvular mitral reumática, y disfonía secundaria a parálisis del nervio laríngeo recurrente izquierdo por compresión auricular ${ }^{1}$. En años recientes se han presentado casos sin crecimiento auricular ni estenosis valvular mitral, por lo que se propuso el término síndrome cardiovocal ${ }^{2}$ para referirse a patologías cardiovasculares intrínsecas que producen parálisis del nervio y diferenciarlas de las causas extrínsecas.

Se presenta el caso de un hombre de 48 años con antecedentes de alcoholismo e insuficiencia cardiaca de origen valvular. Consulta relatando un año de disnea NYHA (New York Heart Association) III, ortopnea, disfonía, globus faríngeo, disfagia, episodio sincopal con el esfuerzo y pérdida de peso. Los signos vitales eran normales, tenía distensión yugular a $45^{\circ}$, soplo sistólico en los focos aórtico y aórtico accesorio grado III/VI, abdomen distendido con dolor a la palpación en hipocondrio derecho, reflujo hepatoyugular presente, edema grado II en miembros inferiores y llenado capilar adecuado. El electrocardiograma inicial mostró anormalidad atrial izquierda, hipertrofia ventricular izquierda, retraso de la conducción intraventricular y trastornos secundarios de la repolarización. Los laboratorios revelaron lesión renal aguda, hiperbilirrubinemia indirecta, sin elevación de transaminasas y gases arteriales con acidosis metabólica compensada. La ecografía abdominal reportó ascitis, distensión de la vena cava inferior y suprahepáticas; la ecocardiografía transtorácica (Figura 1) evidenció crecimiento biauricular severo, ventrículo izquierdo con hipertrofia excéntrica, hipocinesia global, fracción de eyección del 17\%, válvula aórtica con estenosis severa por área $(0.45$ $\mathrm{cm}^{2}$ ) y moderada por gradientes y velocidad (probable bajo flujo, bajo gradiente), y una probabilidad alta de hipertensión pulmonar. El servicio de otorrinolaringología realizó nasolaringoscopia, donde encontraron la cuerda vocal izquierda inmóvil en posición paramediana, el esofagograma y tomografía contrastada fue normal. El servicio de cardiología sospechó síndrome de Ortner y solicitó tomografía de tórax y cuello contrastado contrastada (Figura 2), que descartó otras causas de compresión del nervio laríngeo recurrente, pero halló crecimiento de cavidades cardiacas izquierdas y signos de hipertensión pulmonar severos; hallazgos indirectos de compromiso del nervio laríngeo recurrente como desviación anteromedial del cartílago aritenoideo y dilatación del ventrículo laríngeo izquierdo. Se realizó coronariografía, sin observarse lesiones angiográficamente significativas y se llevó a cirugía de reemplazo valvular, donde encuentran válvula aórtica
Correspondencia:

*Daniel Cardona-Correa

E-mail: daniel.cardonac@ipsuniversitaria.com.co
Disponible en internet: 19-05-2021 Arch Cardiol Mex. 2021;91(4):535-538 www.archivoscardiologia.com
Fecha de recepción: 02-02-2021

Fecha de aceptación: 08-04-2021 DOI: 10.24875/ACM.21000043

1405-9940 / @ 2021 Instituto Nacional de Cardiología Ignacio Chávez. Publicado por Permanyer. Este es un artículo open access bajo la licencia CC BY-NC-ND (http://creativecommons.org/licenses/by-nc-nd/4.0/). 


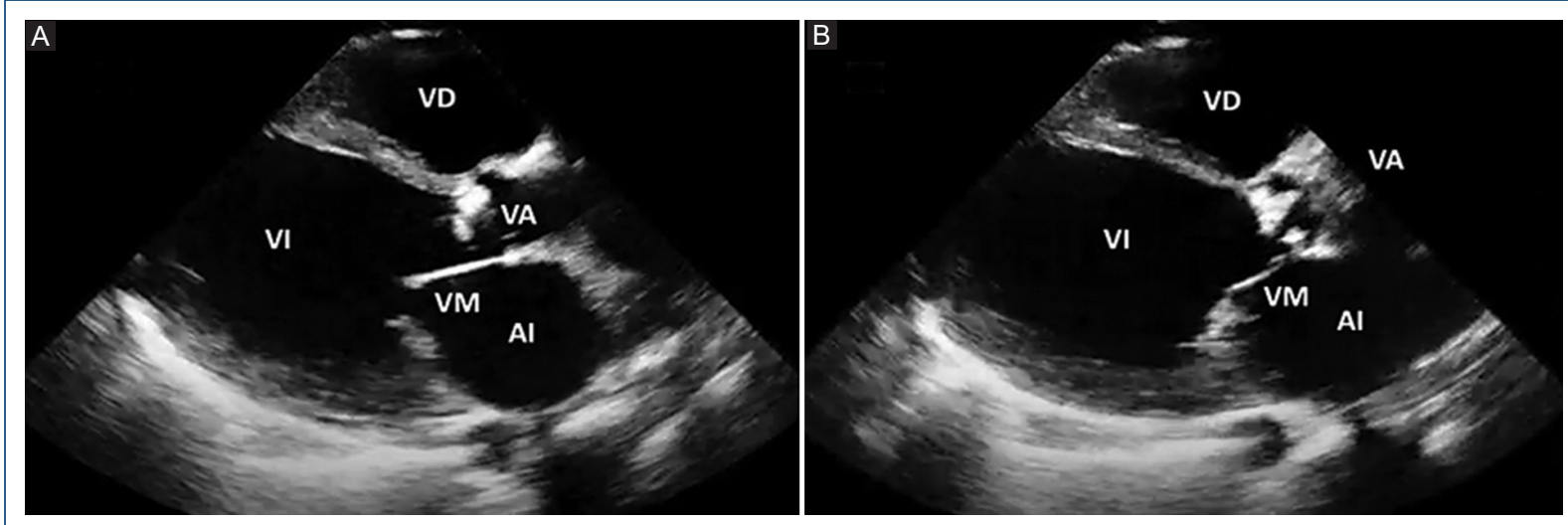

Figura 1. Ecocardiografía transtorácica bidimensional en el plano paraesternal largo, que muestra aparato valvular aórtico con valvas calcificadas y engrosadas, aparato valvular mitral con valvas normales y adecuada movilidad, crecimiento de cavidades izquierdas. A: diástole cardiaca, válvula mitral con adecuada apertura. B: sístole cardiaca, válvula mitral con adecuado cierre.

VI: ventrículo izquierdo; VD: ventrículo derecho; Al: atrio izquierdo; VA: aparato valvular aórtico; VM: aparato valvular mitral.

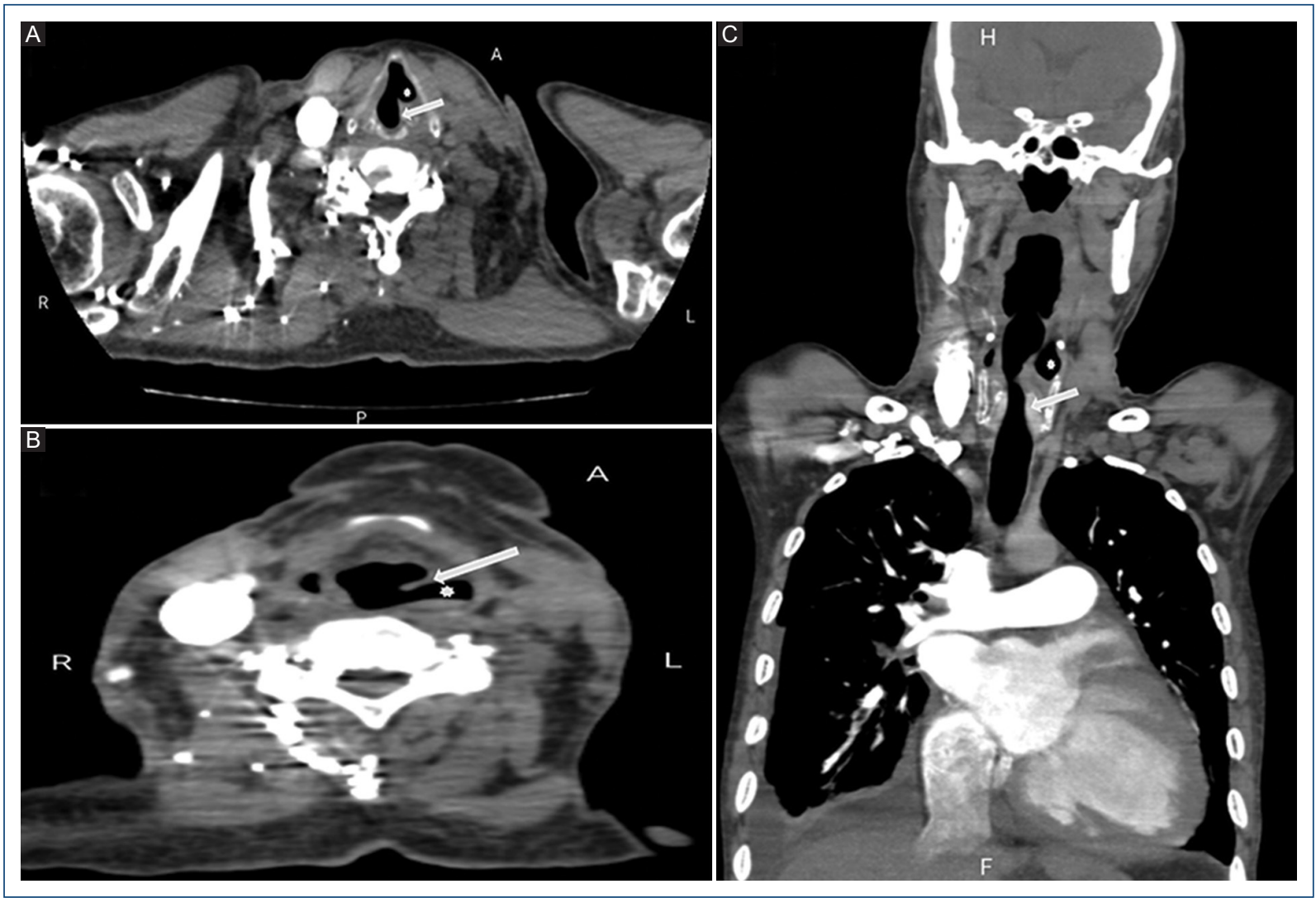

Figura 2. Signos indirectos de parálisis del nervio laríngeo recurrente izquierdo. A: corte axial angiotomografía de cuello. Al nivel de las cuerdas vocales se identifica desplazamiento anterior y medial del cartílago aritenoepiglótico izquierdo (flecha blanca) y dilatación del ventrículo laríngeo izquierdo (asterisco blanco). B: corte axial angiotomografía de cuello a nivel de la hipofaringe. Se evidencia dilatación de la fosa piriforme izquierda (asterisco blanco) y desplazamiento medial y posterior del pliegue aritenoepiglótico izquierdo que se encuentra engrosado (flecha blanca). C: corte coronal angiotomografía de cuello y tórax. Pérdida en la configuración del arco subglótico por debajo de cuerda vocal izquierda por su parálisis (flecha blanca) y dilatación de seno piriforme (asterisco blanco). 
bivalva con anillo aórtico pequeño y realizan reconstrucción de la aorta ascendente e implantación de válvula biológica. En el postoperatorio presentó paro cardiorrespiratorio con actividad eléctrica sin pulso, fue llevado nuevamente a cirugía por sospecha de taponamiento cardiaco y se realizó drenaje mediastinal; posteriormente desarrolló una neumonía asociada al ventilador, falla multiorgánica y muerte por sepsis.

El nervio laríngeo recurrente inerva los músculos intrínsecos ipsilaterales de la laringe a excepción del músculo cricotiroideo, su parálisis condiciona que la cuerda vocal permanezca en posición paramediana produciendo síntomas insidiosos e inicialmente intermitentes. El más frecuente es la disfonía, que puede progresar hasta afonía, disnea durante el discurso y tos por broncoaspiración, especialmente con líquidos ${ }^{2,3}$. Otras manifestaciones se relacionan con la causa de la parálisis e incluyen: dolor torácico no anginoso, claudicación de miembros superiores, disfagia, disnea progresiva o síncopes, en casos de estenosis aórtica ${ }^{2,4}$.

Las etiologías más importantes y más frecuentes son tumores no laríngeos $(40 \%)^{5}$, intervenciones quirúrgicas en cuello $(20 \%)$, trauma (incluyendo intubación orotraqueal) $(10 \%)$ e idiopáticas $(20 \%)^{6}$. El síndrome cardiovocal representa menos del $5 \%$ de las causas ${ }^{4}$.

Desde la descripción de Ortner se han reportado pacientes con disfonía y parálisis de la cuerda vocal izquierda sin evidencia de crecimiento atrial izquierdo ni estenosis valvular mitral, pero con evidencia de compresión del nervio laríngeo recurrente en el triángulo configurado entre el arco aórtico, la arteria pulmonar izquierda y el ligamento arterioso, espacio de tan solo 4 milímetros de diámetro7. Más frecuentemente se afecta el lado izquierdo, favorecido por su ubicación anatómica, ya que luego de su origen en el nervio vago, el tramo intratorácico discurre a través de la ventana aortopulmonar y asciende a lo largo del mediastino y cuello. El mecanismo fisiopatológico aceptado es la lesión mecánica por pinzamiento debido a dilatación de estructuras vasculares como la arteria pulmonar izquierda, en el contexto de hipertensión pulmonar, o el arco aórtico por ensanchamiento aneurismático ${ }^{7}$, pero dada la baja incidencia de la parálisis vocal en estas patologías $(0.5-1 \%)$, se cree que se debe a la suma de varias condiciones: linfadenitis mediastinal, arteria subclavia lusoria, tracción caudal del nervio por ventriculomegalia, compresión directa por el atrio o bronquio fuente izquierdo, inflamación de los tejidos alrededor del nervio, o condiciones que conduzcan a hipertensión pulmonar secundaria: falla ventricular izquierda, cardiopatías congénitas o tromboembolia pulmonar crónica, entre otros.

El diagnóstico se realiza con imágenes que demuestren una condición cardiovascular que explique la parálisis y descarten patologías diferenciales. Es necesaria una laringoscopia para documentar la parálisis de la cuerda vocal y se requieren tomografías de cuello y tórax buscando el sitio de compresión del nervio, hallazgos indirectos como desviación anteromedial del cartílago aritenoideo y dilatación del ventrículo laríngeo izquierdo ${ }^{8}$.

El manejo se basa en la identificación y tratamiento de la causa subyacente, usualmente mediante cirugía ${ }^{3,9}$. El pronóstico es más favorable entre menos tiempo transcurra desde el inicio de los síntomas, ya que esto se relaciona con la gravedad de la lesión (neuropraxia o axonotmesis) y la recuperación de la disfonía puede iniciar hasta tres días tras la intervención ${ }^{10}$. Otras alternativas cuando el paciente o la etiología no son susceptibles de cirugía son la rehabilitación vocal y la tiroplastia.

El síndrome de Ortner es una causa poco frecuente de disfonía. A nuestro conocimiento, este es el primer reporte en la literatura que muestra una estenosis severa de la válvula aórtica como causa de lesión del nervio laríngeo recurrente. Planteamos entonces que esta valvulopatía, con el crecimiento de cavidades izquierdas y la hipertensión pulmonar secundarias, pueden ocasionar la tracción y lesión del nervio.

En conclusión, vale la pena incluir algunas patologías cardiacas, incluyendo la estenosis aórtica severa, dentro del diagnóstico diferencial de la parálisis de cuerdas vocales y lesiones en los nervios laríngeos recurrentes.

\section{Financiamiento}

La presente investigación no ha recibido ayudas específicas provenientes de agencias del sector público, sector comercial o entidades sin ánimo de lucro.

\section{Conflicto de intereses}

Los autores declaran no tener conflicto de intereses.

\section{Responsabilidades éticas}

Protección de personas y animales. Los autores declaran que para esta investigación no se han realizado experimentos en seres humanos ni en animales. 
Confidencialidad de los datos. Los autores declaran que han seguido los protocolos de su centro de trabajo sobre la publicación de datos de pacientes.

Derecho a la privacidad y consentimiento informado. Los autores han obtenido el consentimiento informado de los pacientes y/o sujetos referidos en el artículo. Este documento obra en poder del autor de correspondencia.

\section{Bibliografía}

1. Ortner N. Recurrenslahmung bei Mitralstenose. Wien Klin Wschr [Internet]. 1897;10:753-62. Disponible en: https://ci.nii.ac.jp/naid/10018400808/

2. Kheok SW, Salkade PR, Bangaragiri A, Koh NSY, Chen RC. Cardiovascular hoarseness (Ortner's Syndrome): A pictorial review. Curr Probl Diagn Radiol. 2020 Sep 23;S0363-0188(20)30190-0. doi: 10.1067/j.cpradiol.2020.09.015. Online ahead of print.
3. Tan TXZ, Balakrishnan T, Lam MHH, Chui YY, Cheng LTE. A case of hoarseness with acute back pain-cardiovocal syndrome revisited. J Radiol Case Rep. 2019;13(7):21-8.

4. Mulpuru SK, Vasavada BC, Punukollu GK, Patel AG. Cardiovocal syndrome: A systematic review. Hear Lung Circ. 2008;17(1):1-4.

5. Klee K, Eick C, Witlandt R, Gawaz M, Didczuneit-Sandhop B. Unilateral recurrent nerve palsy and cardiovascular disease-Ortner's syndrome. J Cardiol Cases. 2017;15(3):88-90

6. Rosenthal LHS, Benninger MS, Deeb RH. Vocal fold immobility: A longitudinal analysis of etiology over 20 years. Laryngoscope. 2007;117(10):1864-70.

7. Fetterolf $\mathrm{G}$. The anatomical explanation of the paralysis of the left recurrent laryngeal nerve found in certain cases of mitral stenosis. Am J Med Sci. 1911;331(1):625-39.

8. Paquette CM, Manos DC, Psooy BJ. Unilateral vocal cord paralysis: A review of $C T$ findings, mediastinal causes, and the course of the recurrent laryngeal nerves. Radiographics. 2012;32(3):721-40.

9. Yuan SM. Hoarseness due to aortic arch aneurysms. Brazilian J Cardiovasc Surg. 2020;35(6):970-6

10. Wu VC-C, Chen C-C, Hung K-C, Chern M-S, Wan Y-L, Tsai F-C, et al. Reversal of hoarseness with recognition of Ortner syndrome in a patient with severe mitral regurgitation. J Cardiol Cases. 2012;7(2): e48-e50. 